\title{
Persistent deficiency of mucosa-associated invariant T (MAIT) cells during alcohol-related liver disease
}

\author{
Yujue Zhang ${ }^{1 \dagger}$, Yuanyuan Fan ${ }^{2 \dagger}$, Wei He ${ }^{3}, \mathrm{Yi} \mathrm{Han}^{4}$, Huarui Bao ${ }^{5}$, Renjun Yang ${ }^{1}$, Bingbing Wang ${ }^{1}$, \\ Derun Kong ${ }^{1,4^{*}}$ and Hua Wang ${ }^{2,6^{*}}$ (D)
}

\begin{abstract}
Background: Alcohol-related liver disease (ALD) is a major cause of chronic liver diseases. Inflammatory response is a basic pathological feature of ALD. Mucosal-associated invariant T(MAIT) cells are a novel population of innate immune cells, which may be depleted in various inflammatory diseases. However, the changes of MAIT cell in ALD remains unclear.
\end{abstract}

Results: In this study, the levels of MAIT cell were significantly decreased in patients with alcoholic fatty liver disease, alcoholic cirrhosis, and mixed cirrhosis (alcoholic + viral). Furthermore, the reduction of circulating MAIT cells was correlated with liver function in patients with cirrhosis. Functional changes among circulating MAIT cells in patients with alcoholic cirrhosis, including increased production of IL-17A and perforin, and reduced production of TNF-a. Plasma cytokine and chemokine levels were quantified using multiple immunoassays and ELISA. Serum levels of chemokine IL-8 were correlated with MAIT cell frequency in patients with alcoholic cirrhosis. Moreover, no differences were observed in the expression of CCR6, CXCR6, and PD-1 in circulating MAIT cells of patients with alcoholic cirrhosis. The MAIT cells in patients with alcoholic cirrhosis were prone to apoptosis, which was promoted by IL-12, IL-18, and IL-8.

Conclusions: Our findings indicate persistent MAIT cell loss during alcohol-related liver disease and suggest that MAIT cells can be promising indicator and therapeutic targets in ALD.

Keywords: Mucosal-associated invariant T cells, Alcohol-related liver disease, Severity, Inflammation, Apoptosis

\section{Introduction}

Alcohol-related liver disease (ALD) is a major cause of chronic liver diseases worldwide. Its recent prevalence in China is similar to that in Europe and the United States [1]. Excess alcohol consumption can increase an individual's susceptibility to viral infection and promote viral replication in vivo, thus promoting the occurrence of liver

\footnotetext{
*Correspondence: kongderun168@163.com; wanghua@ahmu.edu.cn ${ }^{\dagger} Y u j u e$ Zhang and Yuanyuan Fan have contributed equally to this work 1 Department of Gastroenterology, the First Affiliated Hospital of Anhui Medical University, Hefei 230032, China

${ }^{2}$ Department of Oncology, the First Affiliated Hospital of Anhui Medical University, Hefei 230032, Anhui, China

Full list of author information is available at the end of the article
}

cirrhosis and liver cancer [2-4]. Moreover, binge drinking has gradually spread and become popular among young people [5]. The pathophysiology of ALD includes inflammation, gut dysbiosis, poor liver regeneration and immune dysfunction [6]. Immune cells, parenchymal cells, cytokines, and chemokines are involved in the inflammatory responses associated with ALD. Immune monitoring during alcohol consumption is of great significance for disease progression. Thus, it is imperative to better understand the pathogenesis of inflammatory responses in ALD to develop novel diagnostic and therapeutic targets.

Mucosal-associated invariant $\mathrm{T}$ (MAIT) cells are innate immune cells that are abundant in the liver [7]. original author(s) and the source, provide a link to the Creative Commons licence, and indicate if changes were made. The images or other third party material in this article are included in the article's Creative Commons licence, unless indicated otherwise in a credit line to the material. If material is not included in the article's Creative Commons licence and your intended use is not permitted by statutory regulation or exceeds the permitted use, you will need to obtain permission directly from the copyright holder. To view a copy of this licence, visit http://creativecommons.org/licenses/by/4.0/. The Creative Commons Public Domain Dedication waiver (http://creativeco mmons.org/publicdomain/zero/1.0/) applies to the data made available in this article, unless otherwise stated in a credit line to the data. 
MAIT cells can be activated by riboflavin metabolites derived from microorganism. Furthermore, MAIT cells also can be activated by IL-12, IL-18, IL-15 and IL-23. Therefore, MAIT cells can be considered both non-specific immune and acquired immune cells. MAIT cells reportedly have profibrogenic function [8], tissue repair [9] and antitumor [10] function. The diagnostic and prognostic value of MAIT cells in Type 1 diabetes [11] and cancer [12] have been reported. MAIT cells are also associated with the progression of HBV infection [13].

In ALD, although MAIT cells have anti-bacterial potency, they are depleted in both blood and liver [14]. It is of great importance to clarify the cause of MAIT cell loss for ALD therapy. Meanwhile, further exploration is required to identify specific biomarkers of MAIT cells in ALD. In the present study, we assessed whether MAIT cells could be used to identify patients with ALD at high risk for liver injury. We also explored the potential mechanism of MAIT cell loss associated with inflammatory cytokines and chemokines.

\section{Results}

\section{Characteristics of patients}

The clinical characteristics of patients are summarized in Table 1. Patients with alcoholic cirrhosis (53.6 \pm 15.62 years of age) and those with mixed cirrhosis (52.21 \pm 6.30 years of age) tended to be elderly individuals, while binge drinkers were mainly among the younger age group (28.34 \pm 9.59 years). Moreover, male patients were more likely to drink than female patients. In some alcoholic cirrhosis patients, they still had excessive drinks. Liver biochemistry analyses, including alanine aminotransferase (ALT), aspartate transaminase (AST), total bilirubin (TBIL), $\gamma$-glutamyl transferase (GGT), alkaline phosphatase (ALP), and albumin (ALB), showed alterations in patients with cirrhosis. Interestingly, the proportion of neutrophils in binge drinkers was significantly increased.

Table 1 Clinical characteristics of patients

\begin{tabular}{|c|c|c|c|c|c|c|c|}
\hline \multirow[t]{2}{*}{ Parameter } & \multirow{2}{*}{$\begin{array}{l}\text { Non drinker } \\
\text { Healthy control }\end{array}$} & \multirow{2}{*}{$\begin{array}{l}\text { ALD } \\
\text { Alcoholic fatty } \\
\text { liver disease }\end{array}$} & \multirow[b]{2}{*}{ Alcoholic cirrhosis } & \multirow[b]{2}{*}{ Mixed cirrhosis } & \multicolumn{2}{|c|}{$\begin{array}{l}\text { Non-pathological alcohol } \\
\text { drinker controls }\end{array}$} & \multirow[b]{2}{*}{ HBV liver cirrhosis } \\
\hline & & & & & Binge drinker & HDC & \\
\hline Number & 116 & 4 & 48 & 33 & 34 & 11 & 7 \\
\hline $\begin{array}{l}\text { Alcohol withdrawal } \\
\text { (>1 year) }\end{array}$ & - & 0 & 23 & 33 & - & - & - \\
\hline $\begin{array}{l}\text { Excessive drinks in } \\
\text { the last } 30 \mathrm{~d}\end{array}$ & - & - & 10 & 0 & 34 & 11 & - \\
\hline $\begin{array}{l}\text { Gender } \\
\text { Male (\%) }\end{array}$ & $93(80.2 \%)$ & $3(75 \%)$ & $47(97.9 \%)$ & $33(100 \%)$ & 19(55.9\%) & $11(100 \%)$ & $7(100 \%)$ \\
\hline Age(year) & $48.89 \pm 11.21$ & $40.25 \pm 3.30^{*}$ & $53.6 \pm 15.62$ & $52.21 \pm 6.30$ & $28.34 \pm 9.59^{* *}$ & $51.36 \pm 10.62$ & $51.86 \pm 3.18$ \\
\hline $\mathrm{ALT}(\mathrm{U} / \mathrm{L})$ & $26.3 \pm 30.54$ & $71 \pm 28.57$ & $40.4 \pm 52.42$ & $32.25 \pm 17.73$ & $24.4 \pm 5.77$ & $51.73 \pm 89.31$ & $28.71 \pm 16.04$ \\
\hline $\mathrm{AST}(\mathrm{U} / \mathrm{L})$ & $21.08 \pm 11.43$ & $49 \pm 15.28$ & $59.29 \pm 64.85^{* *}$ & $39.88 \pm 19.14^{* *}$ & $23.6 \pm 5.18$ & $24 \pm 10.68$ & $24.43 \pm 9.03$ \\
\hline TBIL $(\mu \mathrm{mol} / \mathrm{L})$ & $13.76 \pm 5.89$ & $21.63 \pm 15.26$ & $44.34 \pm 49.55^{* *}$ & $21.54 \pm 19.63$ & $8.94 \pm 4.68$ & $16.56 \pm 14.56$ & $19.24 \pm 7.79$ \\
\hline $\mathrm{ALB}(\mathrm{g} / \mathrm{L})$ & $41.75 \pm 3.38$ & $37.08 \pm 9.40$ & $31.63 \pm 4.86^{* *}$ & $32.76 \pm 6.45^{* *}$ & $46.82 \pm 4.03$ & $39.24 \pm 4.98$ & $39 \pm 1.53$ \\
\hline$A L P(U / L)$ & $78.10 \pm 20.21$ & $86.25 \pm 13.57$ & $140.16 \pm 54.89^{* *}$ & $124.63 \pm 49.86^{* *}$ & $66.75 \pm 15.59$ & $78.73 \pm 17.64$ & $83.14 \pm 13.06$ \\
\hline GGT(U/L) & $31.71 \pm 47.43$ & $143.75 \pm 108.69$ & $149.78 \pm 170.36^{* *}$ & $70.16 \pm 59.74^{*}$ & $23.75 \pm 10.99$ & $49.64 \pm 58.96$ & $26.14 \pm 24.65$ \\
\hline PT(s) & $11.70 \pm 1.02$ & $13.1 \pm 0.95$ & $15.80 \pm 3.21$ & $15.39 \pm 3.16$ & - & $11.95 \pm 0.7$ & $12.13 \pm 0.8$ \\
\hline WBC $\left(\times 10^{9} / L\right)$ & $5.8 \pm 1.59$ & $7.87 \pm 2.42$ & $4.83 \pm 2.22$ & $4.0 \pm 4.04$ & $9.8 \pm 3.64^{* *}$ & $6.1 \pm 0.73$ & $4.99 \pm 0.77$ \\
\hline NEUT (\%) & $54.59 \pm 7.57$ & $71.45 \pm 10.58$ & $61.37 \pm 15.98$ & $58.73 \pm 11.27$ & $71.37 \pm 12.45^{* *}$ & $58.6 \pm 8.64$ & $53.03 \pm 7.4$ \\
\hline Neutrophil $\left(\times 10^{9} / \mathrm{L}\right)$ & $3.22 \pm 1.21$ & $5.75 \pm 2.56$ & $3.05 \pm 1.91$ & $2.47 \pm 2.98$ & $7.22 \pm 3.52^{* *}$ & $3.63 \pm 0.86$ & $2.94 \pm 1.2$ \\
\hline $\begin{array}{l}\text { Lymphocyte } \\
\qquad\left(\times 10^{9} / \mathrm{L}\right)\end{array}$ & $2.37 \pm 4.39$ & $1.44 \pm 0.51$ & $1.18 \pm 0.85$ & $0.92 \pm 0.81^{*}$ & $2.11 \pm 0.99$ & $1.87 \pm 0.41$ & $1.85 \pm 0.39$ \\
\hline
\end{tabular}

All values provided as mean \pm SEM; P-valued were determined by the one-way ANOVA(LSD), or Welch's ANOVA test (Games-Howell test)

$A L T$ alanine aminotransferase, $A S T$ aspartate transaminase, TBIL total bilirubin, GGT $Y$-glutamyl transferase, $A L P$ alkaline phosphatase, $A L B$ albumin, $P T$ prothrombin time

All healthy controls $(\mathrm{HCs})(\mathrm{n}=116)$ must exclude drinking history. ALD patients include alcoholic fatty liver disease $(A F L)(n=4)$, alcoholic cirrhosis $(n=48)$. Nonpathological alcohol drinker controls include binge drinkers $(n=34)$ and heavy drinkers without overt clinical evidence of liver disease $(H D C)(n=11)$. Patients with chronic HBV infection $(n=7)$ and mixed cirrhosis $(n=33)$ were HBV DNA-negative.

${ }^{*} \mathrm{p}<0.05,{ }^{* *} \mathrm{p}<0.01$ (compared with blood from healthy individuals) 


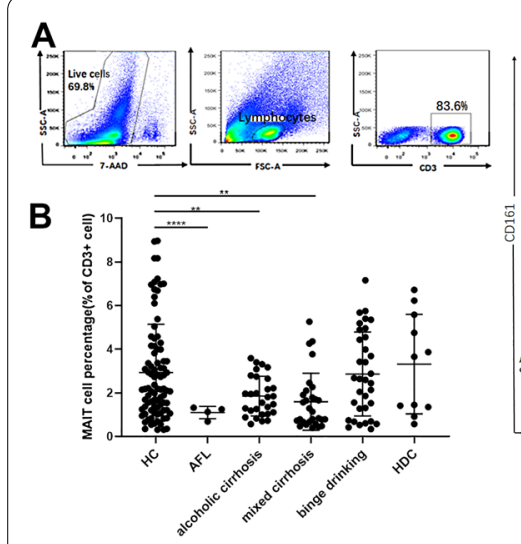

D
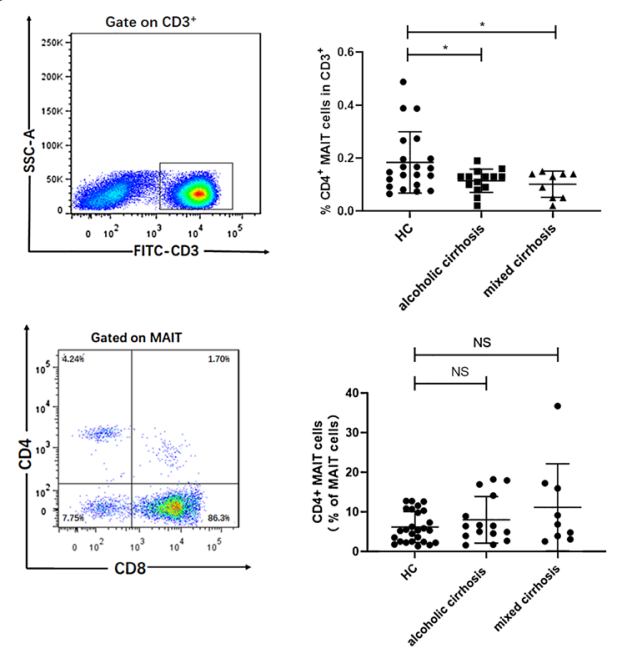

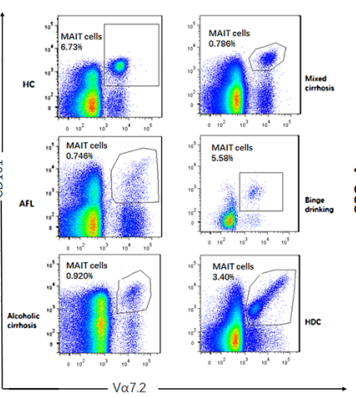

C

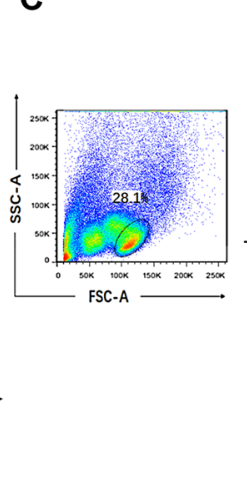

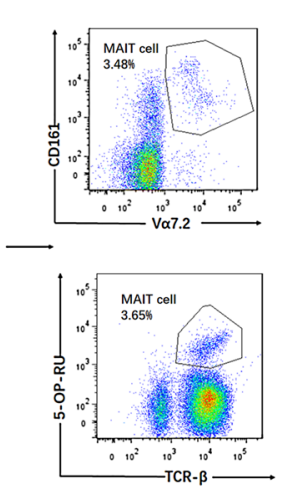

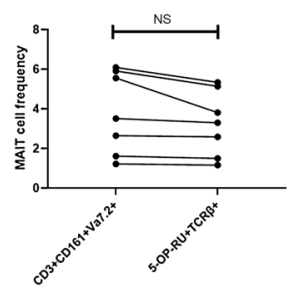

E

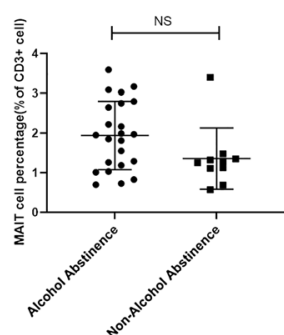

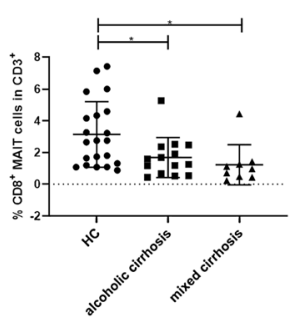
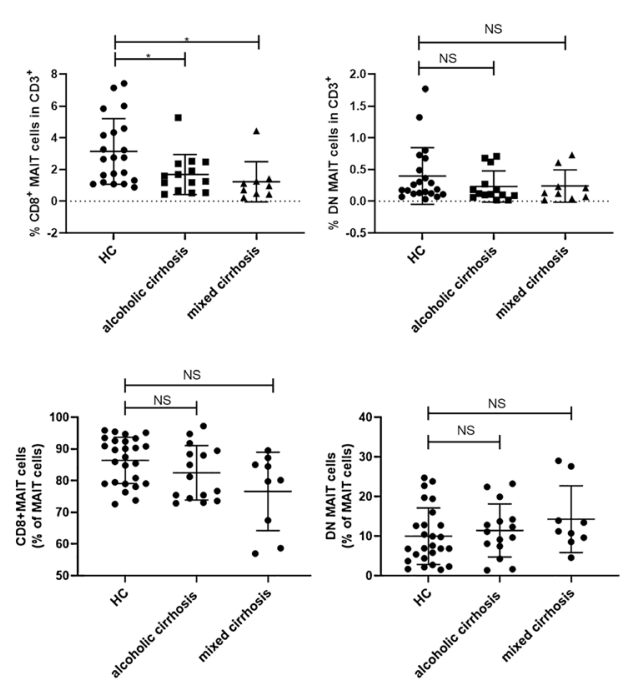

Fig. 1 Circulating MAIT cell frequency was reduced in patients with ALD. A Gating strategy to identify mucosal-associated invariant T (MAIT) cells. Flow cytometry analysis of MAIT cells in human blood using $\mathrm{CD}^{+} \mathrm{CD} 161^{+} \mathrm{Va} 7.2^{+}$. B MAlT cells were depleted in patients with alcoholic fatty liver disease $(n=4)$, alcoholic cirrhosis $(n=29)$, mixed cirrhosis $(n=29)$, and in those who were binge drinker $(n=34)$ and heavy drinkers without overt liver diseases $(n=11)$, compared with HCs $(n=88)$. C MAIT cells were stained with the MR1 tetramer and T-cell receptor beta (TCR- $\beta$ ). No significant differences were observed between the two strategies $(p=0.065)$. D Percentage and constituent ratio of $\mathrm{CD}^{+}, \mathrm{CD}^{+}$, and double negative (DN) MAIT cells in patients with alcoholic cirrhosis, mixed cirrhosis, and HCs. E No significant differences were observed in MAIT cell frequency in alcoholic cirrhosis patients, who either did $(n=23)$ or did not $(n=10)$ abstain from alcohol consumption. Data are presented as the Mean \pm SEM and analyzed by the paired-sample $t$-test, two-sample $t$-test, Welch's analysis of variance (ANOVA) test, ANOVA test, LSD test, and Games-Howell test. $H C$, healthy control; HDC, heavy drinking controls $\left({ }^{*} p<0.05 ;{ }^{* *} p<0.01 ; N S . p>0.05\right)$

\section{Circulating CD4 + and CD8 + MAIT cells are depleted in patients with alcoholic cirrhosis and mixed cirrhosis} Human MAIT cells were gated on $\mathrm{CD}^{+} \mathrm{CD} 161^{+} \mathrm{V} \alpha 7.2^{+}$ lymphocytes (Fig. 1A). The frequency of MAIT cells was significantly depleted in patients with alcoholic fatty liver diseases $(p<0.001)$, alcoholic cirrhosis $(p=0.003)$ and mixed cirrhosis $(\mathrm{p}=0.002)$, compared with HCs (Fig. 1B). However, no obvious reductions in the frequency of MAIT cells were observed among binge drinkers $(p=1)$ and heavy drinkers without overt liver diseases $(p=0.982)$. The frequency of MAIT cells had no difference in patients with alcoholic cirrhosis and mixed cirrhosis $(p=0.903)$. Furthermore, we found a significant reduction in the frequency of MAIT cells in patients with chronic HBV infection compared with healthy controls $(p=0.029)$ (Additional file 1: Fig. S1). In addition, no significant differences in the frequency of MAIT cells were observed between patients with chronic HBV infection and patients with mixed cirrhosis. We also used the 5 -OP-RU tetramer and TCR $\beta$ to confirm our gating strategy and found no significant differences between the two gating strategies $(\mathrm{p}=0.065)$ (Fig. 1C).

MAIT cells can be divided into subsets based on CD4 and CD8 expression [15]. In patients with cirrhosis, $\mathrm{CD}^{+}$ and $\mathrm{CD} 4^{+}$MAIT cells among the T cells were both lower compared with HCs. Our further analysis indicated that 
the percentage of $\mathrm{CD} 8^{+}, \mathrm{CD} 4^{+}$, and $\mathrm{CD} 4^{-} \mathrm{CD} 8^{-}$(double negative, DN) cells among total MAIT cells were similar among patients with alcoholic cirrhosis, mixed cirrhosis, and HCs (Fig. 1D). In addition, no significant associations were noted between gender $(p=0.656)$ or age $(p=0.504)$ and MAIT cell frequency in HCs (Additional file 2: Fig. S2). We found there are no significant changes in the frequency of MAIT cells in alcoholic cirrhosis patients who had abstained from alcohol over 1 year, compared with patients who had still been excessive drinking $(p=0.076)$ (Fig. 1E). Thus, MAIT cells are depleted in chronic liver diseases, possibly related to long-term diseased state.

\section{Correlation between MAIT cell frequency and liver function in patients with cirrhosis}

To assess the clinical significance of reduced MAIT cells in patients with alcoholic cirrhosis and mixed cirrhosis, we performed Spearman's correlation analysis. Based on changes in clinical parameters presented in Table 1, we gained further insights regarding AST,
GGT, TBIL, ALP, and albumin levels. Notably, a negative correlation was found between MAIT cell frequency and AST $(\mathrm{p}=0.02)$, GGT $(\mathrm{p}<0.001)$, and TBIL $(\mathrm{p}=0.004)$ levels in patients with alcoholic cirrhosis, while no correlation was founded between ALB levels $(p=0.747)$ (Fig. 2A). In addition, a negative correlation was noted between MAIT cell frequency and AST, TBIL in all patients with cirrhosis. The correlation between MAIT cell frequency and GGT was not as evident as that in patients with alcoholic cirrhosis (Fig. 2B).

To assess the diagnostic ability of MAIT cells as it relates to validated markers of liver function, we constructed a receiver operating characteristic curve. We evaluated the diagnostic value of MAIT cells for cirrhosis, and determined the area under the curve, sensitivity, specificity, and cut-off values to be $0.703,49.4 \%$, $83.1 \%$, and $2.805 \%$, respectively (Fig. $2 \mathrm{C}$ ). In order to discriminate between patients with cirrhosis and ChildPugh A or Child-Pugh B or, C, we constructed a ROC

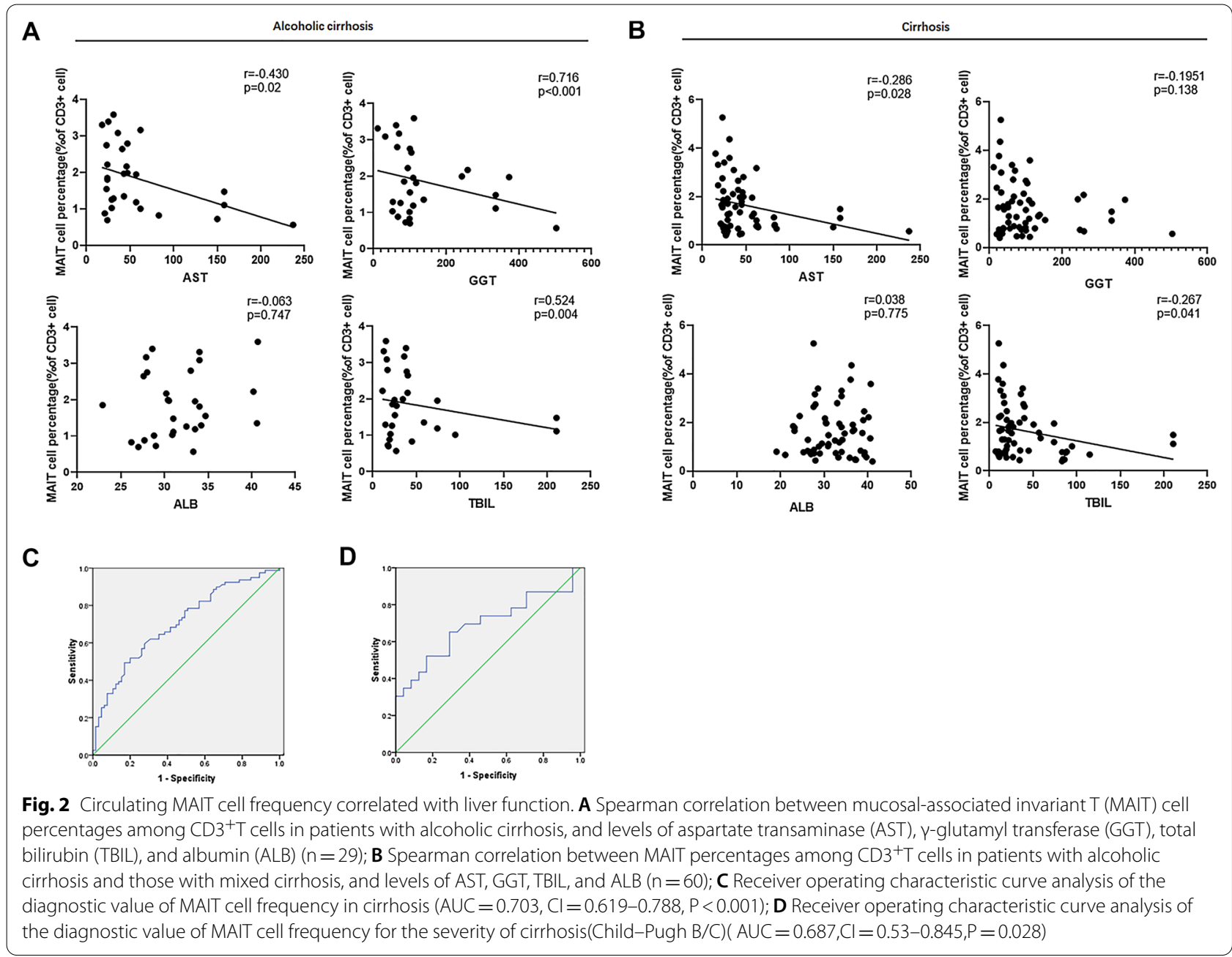


characteristic, which yielded an area under the curve, sensitivity, specificity and cut-off values of $0.687,65.2 \%$, $70.8 \%$, and $1.515 \%$, respectively (Fig. 2D). Taken together, our data reveal a negative correlation between circulating MAIT cell frequency and liver function, and support the notion that MAIT can be a biomarker to evaluate liver injury in ALD.

\section{Activated MAIT cells display an altered cytokine profile} and cytotoxic ability in patients with alcoholic cirrhosis We investigated the phenotype and effector functions of circulating MAIT cells in HCs and patients with ALD. We determined that circulating MAIT cells were activated in patients with cirrhosis by the elevation of CD69 levels (Fig. 3A). As $\mathrm{T}$ cell activation is associated with a

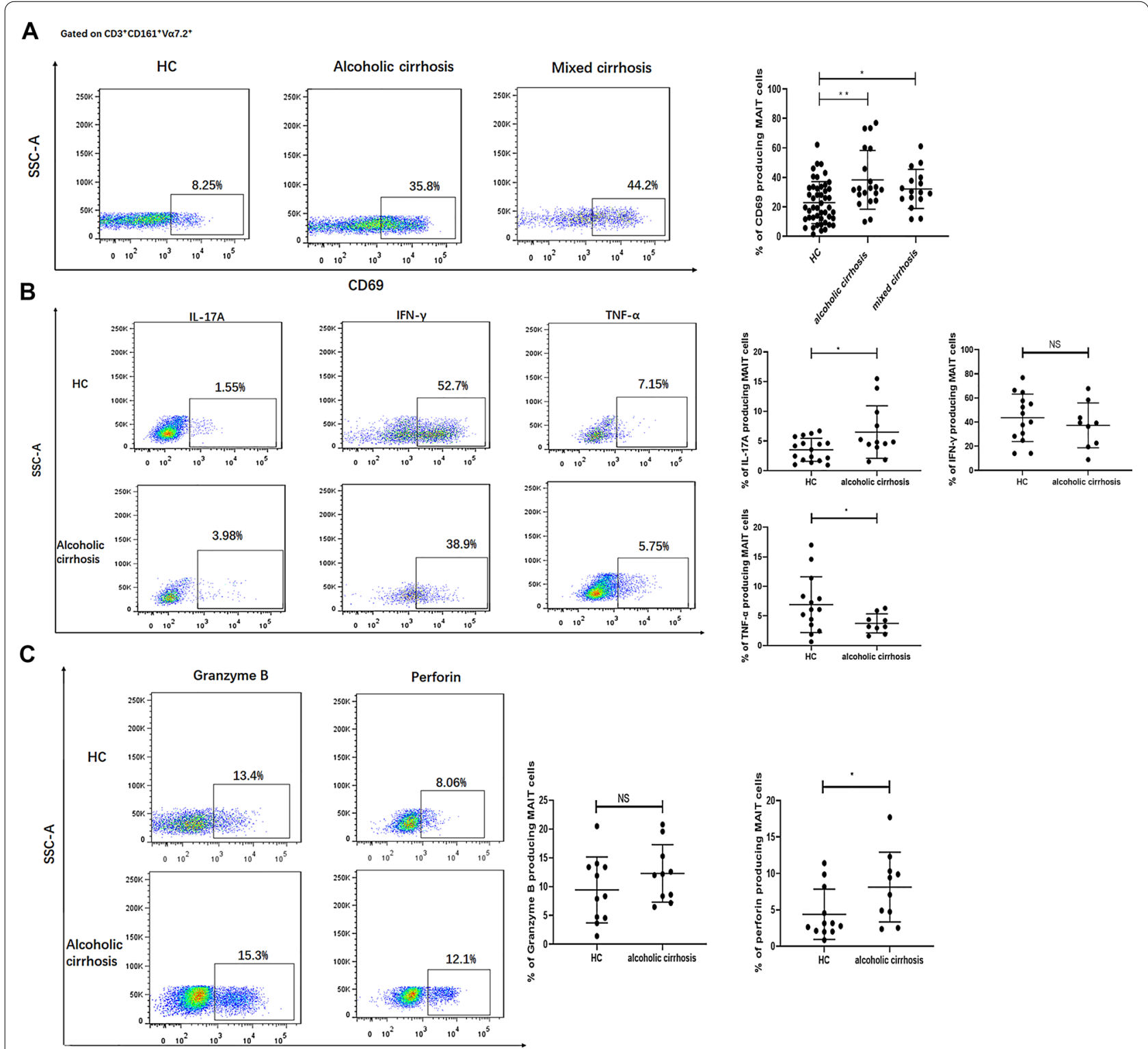

Fig. 3 Phenotype and function of circulating MAIT cells in patients with alcoholic and mixed cirrhosis compared with healthy controls. A Representative dot plot of CD69. Comparison of CD69 on peripheral mucosal-associated invariant T (MAIT) cells in alcoholic liver disease ( $n=20$ ), mixed cirrhosis $(n=16)$, and healthy control $(n=47)(F=7.418, p=0.001)$. B Representative intracellular cytokine staining of MAlT cells from healthy control (HCs) and patients with alcoholic cirrhosis. Comparison of IL-17A, IFN- $\gamma$, TNF-a levels between HC $(n=14-17)$ and patients with alcoholic cirrhosis $(n=9-12)$. C Cytolytic capacity profile of MAIT cells in the blood from HCs and patients with alcoholic cirrhosis. Comparison of Granzyme $B$ and perforin between $\mathrm{HC}(n=11-12)$ and alcoholic cirrhosis $(n=10-11)$. Data were analyzed using the analysis of variance (ANOVA) test, LSD test, and two-sample t-test. $\left({ }^{*} p<0.05 ;{ }^{* *} p<0.01 ;\right.$ NS. $\left.p>0.05\right)$ 
change in effector function, we analyzed the ability of MAIT cells to produce cytokines and cytolytic proteins after PMA-ionomycin stimulation. Compared with HCs, IL-17 production was elevated in MAIT cells of patients with alcoholic cirrhosis $(\mathrm{p}=0.02)$. The MAIT cells produced markedly lower levels of TNF- $\alpha$ in patients with alcoholic cirrhosis $(\mathrm{p}=0.033)$. Moreover, MAIT cells showed higher perforin levels in patients with alcoholic cirrhosis $(\mathrm{p}=0.046)$. In contrast, IFN- $\gamma$ and granzyme B production were similar among MAIT cells in the blood samples of all subjects (Fig. 3B, 3C). Taken together, MAIT cells may be highly activated in patients with alcoholic cirrhosis, which results in an altered cytokine profile and cytotoxic ability.
Elevated IL-8 levels combined with elevated IL-12 and IL-18 levels promote apoptosis of MAIT cells in vivo and in vitro We tried to determine the underlying cause for the reduced circulating MAIT cell frequency in patients with ALD. Previous studies have demonstrated that MAIT cells may be recruited to the liver in chronic liver diseases, which can be identified by elevated levels of CCR6 and CXCR6 [15]. However, we observed no increase in levels of CCR6 and CXCR6 on circulating MAIT cells in patients with alcoholic and mixed cirrhosis (Fig. 4A). Furthermore, previous studies have shown that apoptosis [17] or exhaustion $[18,19]$ may lead to the reduction in MAIT cell frequency. The expression of the immune checkpoint inhibitory molecule PD-1, often indicates $\mathrm{T}$

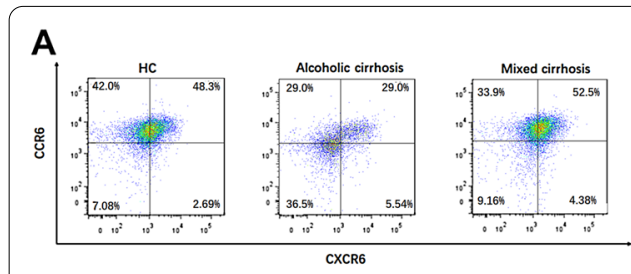

B

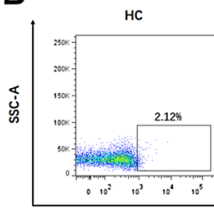

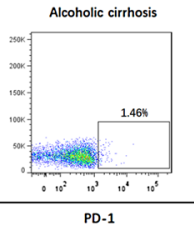

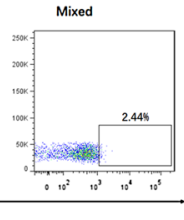

D
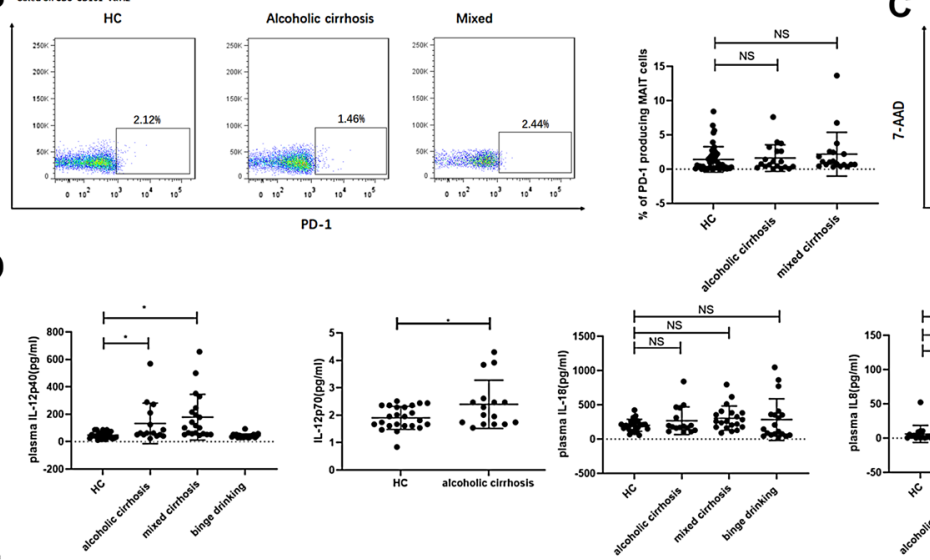

C
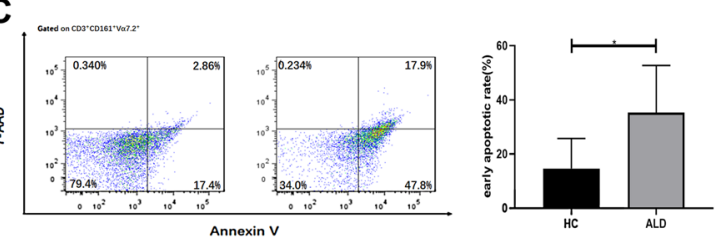

E
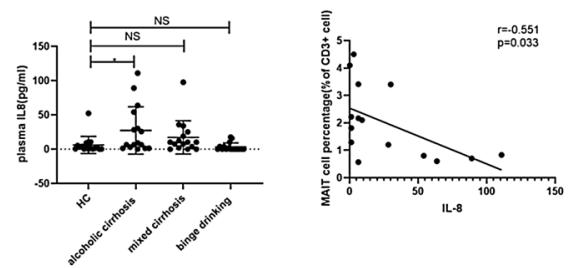

$\mathbf{F}$
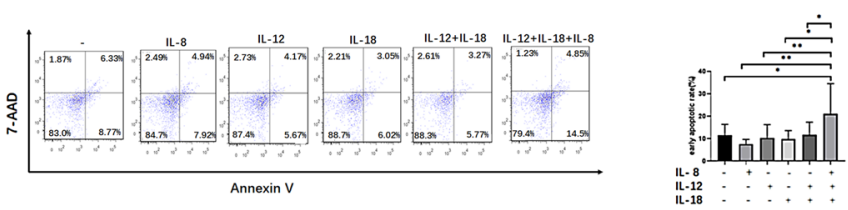

Fig. 4 Inflammatory cytokines and chemokines promoted MAIT cell apoptosis. A Representative dot plot of CCR6 and CXCR6. Comparison of CCR6 and CXCR6 on peripheral mucosal-associated invariant T (MAIT) cells in alcoholic liver disease $(n=18)$, mixed cirrhosis $(n=19)$ and healthy control $(n=48)$. B Representative dot plot of PD-1. Comparison of PD-1 on peripheral MAlT cells in alcoholic liver disease $(n=19)$, mixed cirrhosis $(n=19)$ and healthy control $(n=48)(F=0.816, p=0.446)$. C Peripheral blood mononuclear cells (PBMCs) from patients with alcoholic cirrhosis $(n=5)$ and healthy controls $(n=6)$ were gated on MAIT cells and then stained with 7-aminoactinomycin D (7-AAD) and Annexin V. Percentage of early apoptotic (7-AAD-Annexin $\mathrm{V}^{+}$) cells were measured in healthy controls and patients with alcoholic cirrhosis. D Levels of IL-12p40, IL-12p70, and IL-8 were elevated in patients with alcoholic cirrhosis $(n=16)$ compared with healthy controls $(n=18-24)$. IL-18 was highly expressed in all groups. $\mathbf{E}$ Spearman correlation between MAIT percentage among $\mathrm{CD}^{+}{ }^{+}$T cells in patients with alcoholic cirrhosis with IL-8 levels $(n=15)$; $\mathbf{F}$ Representation of gating strategy showing 7-AAD and Annexin V after gating on MAIT cells in PBMCs from healthy humans, which were cultured with $50 \mathrm{ng} / \mathrm{ml} \mathrm{IL}-12$ or/ and IL-18 or/and IL-8 for $24 \mathrm{~h}$. Early apoptosis was evidenced by the percentages of 7-AAD-Annexin $\mathrm{V}^{+}$cells. Induced apoptosis in MAIT cells after exposure to IL-12, IL-18, and IL-8 stimulation for $24 \mathrm{~h}(\mathrm{n}=5)$. Data were analyzed by using the analysis of variance (ANOVA) test, two-sample $t$-test. $\left({ }^{*} p<0.05 ;{ }^{* *} p<0.01 ; N S . p>0.05\right)$ 
cell exhaustion $[15,19]$. However, PD-1 expression on circulating MAIT cells showed no significant differences between patients with cirrhosis and HCs (Fig. 4B). As shown in Fig. 4C, MAIT cells were prone to apoptosis in patients with alcoholic cirrhosis $(\mathrm{p}=0.034)$.

Cytokine-dependent pathways may contribute to MAIT loss [18, 20]. We further quantified cytokines and chemokines in HCs, patients with alcoholic cirrhosis and mixed cirrhosis, as well as binge drinkers (Table 2). As shown in Table 2, IL-12 and IL-23, which activate MAIT cells, were significantly increased in patients with cirrhosis. The concentration of IL-18, which is associated with the activation and exhaustion of MAIT cells [21, 22 ], was considerably elevated in all groups. In patients with alcoholic cirrhosis, the levels of IL- 8 were significantly increased compared with those in HCs (Fig. 4D). Moreover, MAIT cells were inversely correlated with the levels of circulating IL-8 in patients with alcoholic cirrhosis (Fig. 4E). The PBMCs of healthy subjects were exposed to increasing doses of IL-8 and subjected to $24 \mathrm{~h}$ of culture. We found that different levels of IL-8 did not promote apoptosis in MAIT cells (Additional file 3: Fig. S3). Furthermore, we cultured PBMCs from HCs with IL-12, IL-18 and IL-8. Our results indicated that IL-12 and IL-18, combined with IL-8 can significantly enhance apoptosis of MAIT cells (Fig. 4F). Collectively, these data suggested that the inflammatory environment in alcoholic cirrhosis could promote the apoptosis of MAIT cells.

\section{Discussion}

MAIT cells protect against inflammation and infection in ALD by producing cytokines and cytotoxic effector molecules. Persistent and systemic inflammation may contribute to apoptosis of MAIT cells. To our knowledge, few researches focused on feasibility of MAIT cells as biomarkers for the severity of liver injury in ALD. Our findings show the potential role of MAIT cells in alcoholrelated liver diseases and reveal a possible apoptosisrelated mechanism of MAIT cell loss.

The activation of innate immunity and adaptive immunity contributes to liver inflammation and injury in ALD. MAIT cells are one of important lymphocyte subsets, which are considered to be an important bridge between innate and adaptive immunity. In the present study, we found no significant changes in the MAIT cells of binge drinkers or heavy drinkers without liver diseases. Rapid and excessive alcohol consumption had no effect on MAIT cells. Previous studies have confirmed that shortterm alcohol abstinence does not restore the frequency or function of MAIT cells, while long-term alcohol abstinence only partially reverses abnormalities [14, 18]. Due to the limited samples, we have not identified whether alcohol consumption could affect MAIT cell function and this does require more in-depth investigations.

In the present study, alcohol abstinence had no effect on MAIT cells in patients with ALD. In addition, we found no evidence that the dual factors of a virus and alcohol lead to more severe conditions than alcohol alone

Table 2 Cytokine and chemokine concentrations

\begin{tabular}{|c|c|c|c|c|}
\hline Parameter & $\begin{array}{l}\mathrm{HC} \\
(n=18-24)\end{array}$ & $\begin{array}{l}\text { Alcoholic cirrhosis } \\
(n=16)\end{array}$ & $\begin{array}{l}\text { Mixed cirrhosis } \\
(\mathrm{n}=16-20)\end{array}$ & $\begin{array}{l}\text { Binge drinking } \\
(n=16-20)\end{array}$ \\
\hline TSLP (pg/ml) & $5.77 \pm 3.48$ & $5.64 \pm 4.89$ & $2.11 \pm 0.69^{* *}$ & $1.79 \pm 0.78^{* *}$ \\
\hline IL-23 (pg/ml) & $4.88 \pm 2.49$ & $9.47 \pm 12.34^{*}$ & $9.62 \pm 6.27^{*}$ & $4.27 \pm 1.51$ \\
\hline IL-12p40 (pg/ml) & $50.21 \pm 24.02$ & $139.10 \pm 151.39^{*}$ & $178.98 \pm 166.71^{*}$ & $42.46 \pm 14.87$ \\
\hline IL-12p70 (pg/ml) & $1.90 \pm 0.42$ & $2.15 \pm 0.96^{*}$ & - & - \\
\hline IL-15 (pg/ml) & $17.37 \pm 23.80$ & $59.09 \pm 109.97$ & $121.43 \pm 139.51^{*}$ & $10.70 \pm 42.48$ \\
\hline IL-18 (pg/ml) & $201.0 \pm 85.4$ & $267.37 \pm 201.57$ & $304.48 \pm 177.26$ & $283.61 \pm 303.28$ \\
\hline IL-8 (pg/ml) & $3.22 \pm 3.74$ & $27.30 \pm 34.52^{*}$ & $17.13 \pm 24.24$ & $3.28 \pm 5.83$ \\
\hline CXCL10 (pg/ml) & $58.26 \pm 29.22$ & $126.24 \pm 96.36^{*}$ & $77.40 \pm 37.89$ & $13.57 \pm 9.64^{* *}$ \\
\hline CXCL5 (pg/ml) & $111.18 \pm 168.80$ & $15.74 \pm 21.58^{*}$ & $16.45 \pm 25.73^{*}$ & $82.79 \pm 123.30$ \\
\hline CXCL11 (pg/ml) & $50.51 \pm 27.56$ & $59.16 \pm 33.70^{*}$ & $31.58 \pm 13.08$ & $18.48 \pm 6.87^{* *}$ \\
\hline IL-7 (pg/ml) & $222.01 \pm 46.40$ & $247.52 \pm 43.39$ & $119.58 \pm 21.01^{* *}$ & $175.22 \pm 42.59^{*}$ \\
\hline IL-26 (pg/ml) & $324.42 \pm 122.10$ & $240.47 \pm 115.20$ & $609.6 \pm 177.29^{* *}$ & $485.3 \pm 160.26^{* *}$ \\
\hline CCL11 (pg/ml) & $50.51 \pm 27.56$ & $59.16 \pm 33.70$ & $31.58 \pm 13.08$ & $18.48 \pm 6.87^{*}$ \\
\hline CCL2 (pg/ml) & $294.88 \pm 245.48$ & $260.48 \pm 169.80$ & $152.33 \pm 71.46^{*}$ & $99.41 \pm 109.09^{*}$ \\
\hline CXCL9 (pg/ml) & $26.89 \pm 30$ & $42.51 \pm 55.05$ & $29.70 \pm 29.51$ & $6.14 \pm 9.83^{*}$ \\
\hline
\end{tabular}

All values presented as the mean \pm SEM. p-values were determined by the one-way analysis of variance (ANOVA) least significant difference (LSD), or Welch's ANOVA test (Games-Howell test)

HC healthy controls, TSLP thymic stromal lymphopoietin, IL, interleukin, $C X C L$ chemokine (C-X-C motif) ligand, $C C L$ chemokine (C-C motif) ligand

${ }^{*} \mathrm{p}<0.05,{ }^{* *} \mathrm{p}<0.01$, (compared with blood samples from healthy controls) 
on MAIT cell frequency. The changes in MAIT cells may have been related to persistent pathological changes associated with various diseased states. As a result, a significant reduction in MAIT cells was observed in patients with alcoholic fatty liver diseases, alcoholic cirrhosis and mixed cirrhosis. Furthermore, the frequency of MAIT cells was negatively correlated with AST and TBIL levels in patients with cirrhosis. Specifically, a negative correlation was noted between MAIT cells and GGT in patients with alcoholic cirrhosis. Although the sensitivity of MAIT cells as an initial screening tool is relatively low based on our ROC analysis, the circulating MAIT cell frequency can distinguish HCs from patients with cirrhosis. Moreover, MAIT cells could be used as indicators of the severity of liver disease. Further research will be required to establish the clinical utility of MAIT cells as markers for advanced liver diseases.

CD69 is an immune activation marker which promotes cell proliferation, cytokine production and cytotoxicity [23]. The expression of CD69 on MAIT cells was up regulated in ALD patients. Activated MAIT cells led to altered functions in patients with ALD. Many studies have confirmed that drug therapy could affect MAIT cell frequency and phenotype [8, 15]. Although the changes in circulating MAIT cells were not as informative as those in the liver, they did reflect the state of these cells in inflammatory diseases. IL-17 can regulate alcohol induced liver inflammation, fibrosis, bacterial dissemination and tumorigenesis $[24,25]$. TNF- $\alpha$ is one of inflammatory cytokines for ALD development. TNF- $\alpha$ increased in both the circulation and liver of alcoholic hepatitis and have been shown to be associated with disease severity. Drugs for TNF- $\alpha$ depletion may have therapeutic properties in ALD [26]. The present study demonstrated that MAIT cells produce more IL-17A, and less TNF- $\alpha$ in patients with alcoholic cirrhosis. Furthermore, we assessed cytotoxic activity of MAIT cells which was associated with Granzyme B and perforin [27]. Increased perforin levels may indicate an increased antibacterial potency of MAIT cells in patients with alcoholic cirrhosis. In addition, the trends toward reducing levels of IFN- $\gamma$ and increasing levels of Granzyme B may play a protective role in ALD. Altogether, we postulated that MAIT cells may promote anti-inflammatory and antibacterial responses in ALD.

Several factors may be responsible for MAIT cell depletion, including age [28], gender [15], redistribution [16], cell exhaustion and activation-induced cell death. However, we found no significant differences in gender and age in the MAIT cells of HCs. Neither did we observe any elevated expression of CCR6 or CXCR6 on circulating MAIT cells in patients with alcoholic cirrhosis or mixed cirrhosis. Owing to a lack of liver samples, we could not determine whether MAIT cells had migrated to the liver. Recent research has confirmed the migration of MAIT cells to ascitic fluid in patients with decompensated liver cirrhosis [29]. However, the most recent research has reported that MAIT cells could largely proliferate in situ in the infection organ but do not require CXCR6 for accumulation [30]. Further study of the tissue-resident phenotype of MAIT cells in cross-talk between different organs in patients with ALD may yield promising findings. Furthermore, MAIT cells in this cohort of patients with alcoholic cirrhosis and mixed cirrhosis showed no expression of the immune exhaustion marker, PD-1. In line with previous studies [8], we found that apoptosis may be one of reasons of MAIT cell loss in patients with alcoholic cirrhosis, possibly through activation-induced cell death.

In chronic HBV-infected patients, conjugated bilirubin can promote peripheral MAIT cell activation and apoptosis [13]. Bottcher et al. found long-term stimulation with IL-12 and IL-18 promote MAIT cell death in liver tissue [21]. Our data suggested MAIT cell depletion is a consequence of long-term systemic inflammation. MAIT cell activation-associated cytokines IL-12 and IL-23 were considerably increased in alcoholic cirrhosis. Interestingly, we observed high expression of IL-18 in all groups. IL-18 is a marker of hepatic steatosis and regulate the process of metabolic syndrome in NASH [31, 32]. Besides, IL-18 participate in antiviral therapy and profibrogenic process in various chronic liver diseases [21, 33]. Li et al. [18] reported that IL-18 is negatively correlated with MAIT frequency in alcoholic hepatitis. We also observed that levels of IL-8 were elevated in patients with alcoholic cirrhosis and negatively correlated with MAIT cell frequency. However, we found that different doses of IL-8 had no effect on the apoptosis of MAIT cells. Considering the fact that MAIT cells become altered after activation, a lack of IL-8 receptors on activated MAIT cell is plausible. As previously described $[14,15,29]$, we cultured PBMCs from HCs with IL-12, IL-18 and IL-8. We further confirmed that IL-12, IL-18 and a combination with IL-8 could induce apoptosis. The findings of the present study suggest that a cytokine-rich inflammatory environment promotes MAIT cell loss in patients with ALD. Nonetheless, persistent inflammation may be associated with a variety of cytokines and chemokines. It is also possible that additional cytokines besides those under investigation in the present study, are able to affect MAIT cells. We have only confirmed the effects of a few. Meanwhile, MAIT cell depletion may be related to other pathways of cell death. This process requires further investigation. In our study, we could not determine whether MAIT cells are depleted and occurred apoptosis in the liver. In the future research, 
we will make deep research in the liver of alcohol related liver disease.

\section{Conclusions}

Reduced MAIT cells were negatively correlated with liver injury in patients with cirrhosis. MAIT cells could be used as a new indicator for liver injury in ALD. We demonstrated activated MAIT cells have anti-inflammatory and antibacterial effects. Increased levels of inflammatory cytokines and chemokines promoted the apoptosis of MAIT cells, which may be one of causes for MAIT cell loss. Our data highlighted the diagnostic and therapeutic potential of MAIT cells in ALD. Further studies are required to focus on the mechanisms of upregulation of MAIT cell frequency and the restoration of their function.

\section{Materials and methods Study subjects}

This study was approved by the Ethics Committee of Clinical Research of Anhui Medical University. All participants gave informed consent. All blood samples were collected at The First Affiliated Hospital of Anhui Medical University. The study subjects included patients with alcoholic fatty liver disease (AFL) $(\mathrm{n}=4)$, alcoholic cirrhosis $(\mathrm{n}=48)$, mixed cirrhosis $(\mathrm{n}=33)$, as well as those who were binge drinkers $(n=34)$, heavy drinkers without overt clinical evidence of liver disease (HDC) $(n=11)$, and those with chronic HBV infection $(n=7)$ and HCs $(n=116)$. The diagnosis of patients was based on previously described criteria [34-36]. Binge drinkers had rapid and excessive alcohol intake in $2 \mathrm{~h}$ and HDC patients had long-term drink history. AFL patients had short-term alcohol abstinence and mixed cirrhosis patients all had more than 1 year alcohol withdrawal. In addition to the criteria for alcoholic cirrhosis, patients with mixed cirrhosis also had HBV infection. All patients with HBV infection were HBV DNA-negative. Non-inclusion criteria included recent gastrointestinal bleeding, current bacterial infections, and immunomodulatory or antibiotic treatments. All participants with metabolic diseases, immune diseases, or cancer were excluded. All healthy controls must exclude drinking history. Clinical characteristics of the subjects are presented in Table 1.

\section{Blood samples}

Peripheral blood samples were collected in EDTA tubes and separated into plasma and peripheral blood mononuclear cells (PBMCs). Plasma samples were stored at $-80^{\circ} \mathrm{C}$ until further use. The PBMCs were immediately used for Flow cytometric analysis.

\section{PBMC isolation and culture}

The PBMCs were isolated by density gradient centrifugation, using the Ficoll-Hypaque technique ( Solarbio, China), and freshly used for surface and intracellular staining to analyze frequency, phenotype, and function. To detect cytokine production and cytotoxicity markers, PBMCs were cultured in RPMI-1640 medium supplemented with $10 \%$ fetal bovine serum, and stimulated with phorbol 12-myristate-13-acetate(PMA)/ionomycin and Brefeldin A/Monensin (Multi sciences, China) for 4-6 h at $37{ }^{\circ} \mathrm{C}$. Stimulated PBMCs were fixed and permeabilized using a FIX \& PERM Kit (Multi sciences, China) to stain the intracellular markers. The PBMCs were further stimulated with IL-8, IL-12, or IL-18 (Novoprotein Scientific, China) at $50 \mathrm{ng} / \mathrm{mL}$ for $24 \mathrm{~h}$.

\section{Flow cytometric analysis}

Flow cytometry was performed as described using the following antibodies. The anti-CD3-FITC, antiCD161-APC, and anti-TCR Va7.2-Brilliant Violet 421(Biolegend, USA) were used to identify MAIT cells. Anti-CD4-PE/Cy7, anti-CD8-Perp/Cy5.5, anti-CCR6-PE/ Cy7, anti-CXCR6-PE, anti-IL-17A-PE, anti-Granzyme $\mathrm{B}-\mathrm{PE} / \mathrm{Cy} 7$, and anti-Perforin-PE were obtained from Biolegend (USA). Anti-CD69-PE/Cy7, anti-PD-1-PE, antiIFN- $\gamma$-PE/Cy7, anti-TNF- $\alpha-P E$ were obtained from BD (USA). Appropriate isotype control antibodies were used for each staining combination. We used 7-aminoactinomycin $\mathrm{D}$ (7-AAD) (BD Biosciences) staining to identify dead cells. Human MR1 tetramers loaded with a potent MAIT cell ligand-5-OP-RU or 6-FP were gifted from professor Li Bai. Apoptosis was allowed to progress and two channels were used to detect annexin V- FITC and 7-AAD (BD Biosciences) to determine the proportions of apoptotic cells. Data acquisition was achieved on BD FACS Verse system (BD Biosciences), and results were analyzed using FlowJo7.6 analysis software.

\section{Multiplex immunoassays}

Human cytokines and chemokines were identified in the plasma of patients with alcoholic cirrhosis, mixed cirrhosis, as well as binge drinkers and HCs. The cytokines and chemokines (GM-CSF, IFN- $\alpha 2$, IL- $1 \alpha$, IL-1 $\beta$, IL-11, IL12p40, IL-12p70, IL-15, IL-18, IL-23, IL-27, IL-33, TSLP, CCL2/MCP-1, CCL3/MIP-1 $\alpha$, CCL4/MIP-1 $\beta, \quad C C L 5 /$ RANTES, CCL11/Eotaxin, CCL17/TARC, CCL20/ MIP-3 $\alpha$, CXCL1/GRO $\alpha$, CXCL5/ENA-78, CXCL8/IL-8, CXCL9/MIG, CXCL10/IP-10, CXCL11/I-TAC) were analyzed by flow cytometry bead-based immunoassay (LEGENDplex ${ }^{\mathrm{TM}}$ Human Cytokine Panel2/ Human Proinflammatory Chemokine Panel, BioLegend), according to the manufacturer's instructions. Sample acquisition 
was achieved on a FACS Verse system equipped with the FACSuite software (BD Biosciences), and samples were analyzed with the LEGENDplex ${ }^{\mathrm{TM}}$ Data Analysis Software v8.0.

\section{Enzyme-linked immunosorbent assay (ELISA)}

Circulating levels of IL-7 and IL-26 were not available in the above panel. Therefore, these cytokines in the plasma were quantified using enzyme-linked immunosorbent assay (ELISA; Jianglai Biotech, China).

\section{Statistical analysis}

All data were presented as the mean \pm SEM. The parametric Student's $t$-test for paired or unpaired samples as appropriate, or the Mann-Whitney U test, one-way analysis of variance (ANOVA) least significant difference (LSD), Welch's ANOVA test (Games-Howell test), or Spearman's correlation were used to calculate statistical significance. The diagnostic performance of MAIT cells in identifying the severity of disease was assessed by analyzing receiver operating characteristic (ROC) curves. Statistical calculations were performed using the GraphPad Prism 8.0 and SPSS17.0 software. In all tests, $\mathrm{p}<0.05$ was considered statistically significant.

\begin{abstract}
Abbreviations
ALD: Alcohol-related liver disease; HC: Healthy control; HDC: Heavy drinker without clinical evidence of liver disease; MAIT: Mucosal-associated invariant T cells; PBMC: Peripheral blood mononuclear cells; ALT: Alanine aminotransferase; AST: Aspartate transaminase; TBIL:Total bilirubin; GGT: $\gamma$-Glutamyl transferase; ALP: Alkaline phosphatase; ALB: Albumin.
\end{abstract}

\section{Supplementary Information}

The online version contains supplementary material available at https://doi. org/10.1186/s13578-021-00664-8.

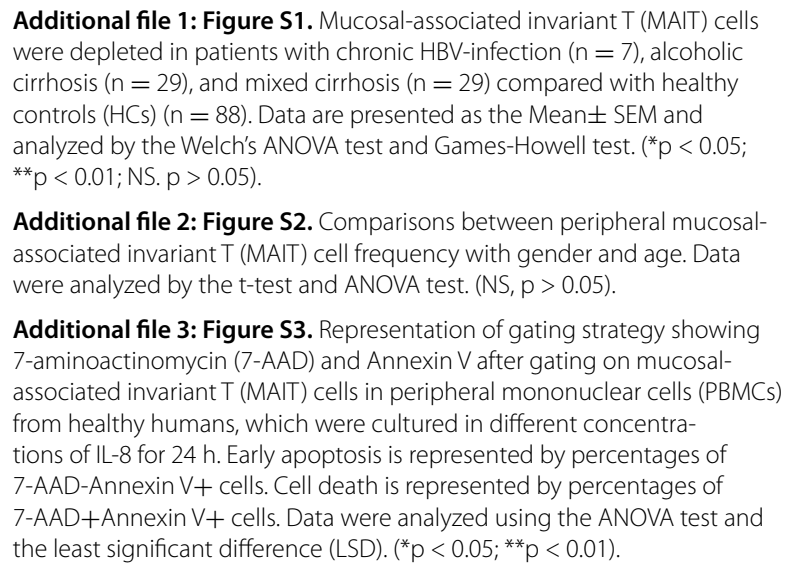
were depleted in patients with chronic HBV-infection $(n=7)$, alcoholic cirrhosis $(n=29)$, and mixed cirrhosis $(n=29)$ compared with healthy controls (HCS) $(n=88)$. Data are presented as the Mean \pm SEM and analyzed by the Welch's ANOVA test and Games-Howell test. $\left({ }^{*} \mathrm{p}<0.05\right.$; ${ }^{* *} \mathrm{p}<0.01$; NS. $p>0.05$ ).

Additional file 2: Figure S2. Comparisons between peripheral mucosalassociated invariant T (MAIT) cell frequency with gender and age. Data were analyzed by the t-test and ANOVA test. (NS, p > 0.05).

Additional file 3: Figure S3. Representation of gating strategy showing 7-aminoactinomycin (7-AAD) and Annexin $V$ after gating on mucosalassociated invariant T (MAIT) cells in peripheral mononuclear cells (PBMCs) from healthy humans, which were cultured in different concentrations of IL-8 for $24 \mathrm{~h}$. Early apoptosis is represented by percentages of 7-AAD-Annexin $V+$ cells. Cell death is represented by percentages of 7-AAD+Annexin V+ cells. Data were analyzed using the ANOVA test and the least significant difference (LSD). $\left({ }^{*} p<0.05\right.$; $\left.{ }^{* *} p<0.01\right)$.

\section{Acknowledgements}

We thank Li Bai, PhD (University of Sciences and Technology of China) for providing us with Human MR1 tetramers, which were loaded with 5-OP-RU and 6-FP.

\section{Authors' contributions}

Experiments were designed by DK and $H W$, and carried out by $Y Z, Y F$ and $W H$. Samples were collected with the help of $Y Z, Y H, H B, R Y$, and BW. The manuscript was written by $Y Z$, and revised by DK and HW. All authors have read and approved the final manuscript.

\section{Funding}

The work was supported by the Research fund project of the Anhui provincial institute of translational medicine [No.2017zhyx18]; and the Anhui Science and Technology Department: 2018 Key research and development plan projects [No.1804h08020260].

\section{Availability of data and material}

All data generated or analyzed during this study are included in this published article.

\section{Declarations}

\section{Ethics approval and consent to participate}

This study was approved by the Medical Ethics Committee of the First Hospital of Anhui Medical University of Anhui Medical University and the methods were carried out in accordance with the approved guidelines.

\section{Consent for publication}

Not applicable.

\section{Competing interests}

The authors declare that they have no competing interests.

\section{Author details}

${ }^{1}$ Department of Gastroenterology, the First Affiliated Hospital of Anhui Medical University, Hefei 230032, China. ${ }^{2}$ Department of Oncology, the First Affiliated Hospital of Anhui Medical University, Hefei 230032, Anhui, China. ${ }^{3}$ School of Basic Medical Sciences, Anhui Medical University, Hefei 230032, Anhui, China. ${ }^{4}$ Department of Gastroenterology, Fuyang Hospital of Anhui Medical University, Fuyang 236000, Anhui, P.R. China. ${ }^{5}$ Department of Emergency, the First Affiliated Hospital of Anhui Medical University, Hefei 230032, China. ${ }^{6}$ Inflammation and Immune Mediated Diseases Laboratory of Anhui Province, Anhui Medical University, Hefei 230032, Anhui, China.

Received: 22 January 2021 Accepted: 20 July 2021

Published online: 28 July 2021

\section{References}

1. Xiao J, Wang F, Wong NK, He J, Zhang R, Sun R, et al. Global liver disease burdens and research trends: analysis from a Chinese perspective. J Hepatol. 2019;71(1):212-21. https://doi.org/10.1016/j.jhep.2019.03.004.

2. Fuster D, Sanvisens A, Bolao F, Rivas I, Tor J, Muga R. Alcohol use disorder and its impact on chronic hepatitis $C$ virus and human immunodeficiency virus infections. World J Hepatol. 2016;8(31):1295-308. https://doi. org/10.4254/wjh.v8.i31.1295.

3. Lin CW, Lin CC, Mo LR, Chang CY, Perng DS, Hsu CC, et al. Heavy alcohol consumption increases the incidence of hepatocellular carcinoma in hepatitis B virus-related cirrhosis. J Hepatol. 2013;58(4):730-5. https://doi. org/10.1016/j.jhep.2012.11.045.

4. Shah ND, Ventura-Cots M, Abraldes JG, Alboraie M, Alfadhli A, Argemi J, et al. Alcohol-related liver disease is rarely detected at early stages compared with liver diseases of other etiologies worldwide. Clin Gastroenterol Hepatol. 2019;17(11):2320-9. https://doi.org/10.1016/j.cgh.2019.01. 026.

5. Mathurin P, Deltenre P. Effect of binge drinking on the liver: an alarming public health issue? Gut. 2009;58(5):613-7. https://doi.org/10.1136/gut. 2007.145573

6. Seitz HK, Bataller R, Cortez-Pinto H, Gao B, Gual A, Lackner C, et al. Alcoholic liver disease. Nat Rev Dis Primers. 2018;4(1):16. https://doi.org/10. 1038/s41572-018-0014-7. 
7. Kurioka A, Walker LJ, Klenerman P, Willberg CB. MAIT cells: new guardians of the liver. Clin TransI Immunol. 2016;5(8):e98. https://doi.org/10.1038/ cti.2016.51.

8. Hegde P, Weiss E, Paradis V, Wan J, Mabire M, Sukriti S, et al. Mucosalassociated invariant $T$ cells are a profibrogenic immune cell population in the liver. Nat Commun. 2018;9(1):2146. https://doi.org/10.1038/ s41467-018-04450-y.

9. Hinks T, Marchi E, Jabeen M, Olshansky M, Kurioka A, Pediongco TJ, et al. Activation and in vivo evolution of the MAIT cell transcriptome in mice and humans reveals tissue repair functionality. Cell Rep. 2019;28(12):3249-62. https://doi.org/10.1016/j.celrep.2019.07.039.

10. Duan M, Goswami S, Shi JY, Wu LJ, Wang XY, Ma JQ, et al. Activated and exhausted mait cells foster disease progression and indicate poor outcome in hepatocellular carcinoma. Clin Cancer Res. 2019;25(11):3304-16. https://doi.org/10.1158/1078-0432.CCR-18-3040.

11. Rouxel O, Da SJ, Beaudoin L, Nel I, Tard C, Cagninacci L, et al. Cytotoxic and regulatory roles of mucosal-associated invariant $T$ cells in type 1 diabetes. Nat Immunol. 2017;18(12):1321-31. https://doi.org/10.1038/ni. 3854 .

12. Li J, Zhou J, Kai S, Wang C, Wang D, Jiang J. Functional and clinical characterization of tumor-infiltrating $\mathrm{T}$ cell subpopulations in hepatocellular carcinoma. Front Genet. 2020;11:586415. https://doi.org/10.3389/fgene. 2020.586415

13. Liu Y, Zhu P, Wang W, Tan X, Liu C, Chen Y, et al. MAIT cell dysregulation correlates with conjugated bilirubin level in chronic hepatitis $B$ virus infection. Hepatology. 2020. https://doi.org/10.1002/hep.31602.

14. Riva A, Patel V, Kurioka A, Jeffery HC, Wright G, Tarff S, et al. Mucosa-associated invariant $T$ cells link intestinal immunity with antibacterial immune defects in alcoholic liver disease. Gut. 2018;67(5):918-30. https://doi.org/ 10.1136/gutjnl-2017-314458.

15. Jiang $X$, Lian M, Li Y, Zhang W, Wang $Q$, Wei Y, et al. The immunobiology of mucosal-associated invariant T cell (MAIT) function in primary biliary cholangitis: regulation by cholic acid-induced Interleukin-7. J Autoimmun. 2018;90:64-75. https://doi.org/10.1016/j.jaut.2018.01.007.

16. Li Y, Huang B, Jiang X, Chen W, Zhang J, Wei Y, et al. Mucosal-associated invariant $T$ cells improve nonalcoholic fatty liver disease through regulating macrophage polarization. Front Immunol. 2018;9:1994. https://doi. org/10.3389/fimmu.2018.01994.

17. Gerart S, Siberil S, Martin E, Lenoir C, Aguilar C, Picard C, et al. Human iNKT and MAIT cells exhibit a PLZF-dependent proapoptotic propensity that is counterbalanced by XIAP. Blood. 2013;121(4):614-23. https://doi.org/10. 1182/blood-2012-09-456095.

18. Li W, Lin EL, Liangpunsakul S, Lan J, Chalasani S, Rane S, et al. Alcohol abstinence does not fully reverse abnormalities of mucosal-associated invariant T cells in the blood of patients with alcoholic hepatitis. Clin TransI Gastroenterol. 2019;10(6): e52. https://doi.org/10.14309/ctg.00000 00000000052.

19. Yong YK, Saeidi A, Tan HY, Rosmawati M, Enstrom PF, Batran RA, et al. Hyper-expression of PD-1 Is associated with the levels of exhausted and dysfunctional phenotypes of circulating CD161(++)TCR iValpha7.2(+) mucosal-associated invariant $\mathrm{t}$ cells in chronic hepatitis B virus infection. Front Immunol. 2018;9:472. https://doi.org/10.3389/fimmu.2018.00472.

20. Touch S, Assmann KE, Aron-Wisnewsky J, Marquet F, Rouault C, Fradet M, et al. Mucosal-associated invariant T (MAIT) cells are depleted and prone to apoptosis in cardiometabolic disorders. Faseb J. 2018. https://doi.org/ 10.1096/f.201800052RR.

21. Bottcher K, Rombouts K, Saffioti F, Roccarina D, Rosselli M, Hall A, et al. MAIT cells are chronically activated in patients with autoimmune liver disease and promote profibrogenic hepatic stellate cell activation. Hepatology. 2018;68(1):172-86. https://doi.org/10.1002/hep.29782.

22. Bolte FJ, O'Keefe AC, Webb LM, Serti E, Rivera E, Liang TJ, et al. Intrahepatic depletion of mucosal-associated invariant T cells in hepatitis $C$ virus-induced liver inflammation. Gastroenterology. 2017;153(5):1392403. https://doi.org/10.1053/j.gastro.2017.07.043.

23. Freeman $B E$, Hammarlund $E$, Raue $H P$, Slifka MK. Regulation of innate CD8+ T-cell activation mediated by cytokines. Proc Natl Acad Sci USA. 2012;109(25):9971-6. https://doi.org/10.1073/pnas.1203543109.

24. Heymann F, Tacke F. Immunology in the liver-from homeostasis to disease. Nat Rev Gastroenterol Hepatol. 2016;13(2):88-110. https://doi. org/10.1038/nrgastro.2015.200.

25. Curtis MM, Way SS. Interleukin-17 in host defence against bacterial, mycobacterial and fungal pathogens. Immunology. 2009;126(2):177-85. https://doi.org/10.1111/j.1365-2567.2008.03017.x.

26. Thursz MR, Richardson P, Allison M, Austin A, Bowers M, Day CP, et al. Prednisolone or pentoxifylline for alcoholic hepatitis. N Engl J Med. 2015;372(17):1619-28. https://doi.org/10.1056/NEJMoa1412278.

27. Makedonas G, Banerjee PP, Pandey R, Hersperger AR, Sanborn KB, Hardy $\mathrm{GA}$, et al. Rapid up-regulation and granule-independent transport of perforin to the immunological synapse define a novel mechanism of antigen-specific CD8+ T cell cytotoxic activity. J Immunol. 2009;182(9):5560-9. https://doi.org/10.4049/jimmunol.0803945.

28. Lee OJ, Cho YN, Kee SJ, Kim MJ, Jin HM, Lee SJ, et al. Circulating mucosalassociated invariant $\mathrm{T}$ cell levels and their cytokine levels in healthy adults. Exp Gerontol. 2014;49:47-54. https://doi.org/10.1016/j.exger.2013. 11.003.

29. Niehaus CE, Strunz B, Cornillet M, Falk CS, Schnieders A, Maasoumy B, et al. MAIT cells are enriched and highly functional in ascites of patients with decompensated liver cirrhosis. Hepatology. 2020. https://doi.org/10. 1002/hep.31153.

30. Yu H, Yang A, Liu L, Mak J, Fairlie DP, Cowley S. CXCL16 stimulates antigeninduced MAIT cell accumulation but trafficking during lung infection is CXCR6-independent. Front Immunol. 2020;11:1773. https://doi.org/10. 3389/fimmu.2020.01773.

31. Flisiak-Jackiewicz M, Bobrus-Chociej A, Tarasow E, Wojtkowska M, Bialokoz-Kalinowska I, Lebensztejn DM. Predictive role of interleukin-18 in liver steatosis in obese children. Can J Gastroenterol Hepatol. 2018;2018:3870454. https://doi.org/10.1155/2018/3870454.

32. Hohenester S, Kanitz V, Schiergens T, Einer C, Nagel J, Wimmer R, et al. IL-18 but not IL-1 signaling is pivotal for the initiation of liver injury in murine non-alcoholic fatty liver disease. Int J Mol Sci. 2020. https://doi. org/10.3390/ijms21228602.

33. Dias J, Hengst J, Parrot T, Leeansyah E, Lunemann S, Malone D, et al. Chronic hepatitis delta virus infection leads to functional impairment and severe loss of MAIT cells. J Hepatol. 2019;71(2):301-12. https://doi.org/10. 1016/j.j.hep.2019.04.009.

34. Li YM, Fan JG, Wang BY, Lu LG, Shi JP, Niu JQ, et al. Guidelines for the diagnosis and management of alcoholic liver disease: update 2010: (published in Chinese on Chinese Journal of Hepatology 2010; 18: 167-170). J Dig Dis. 2011;12(1):45-50. https://doi.org/10.1111/j.1751-2980.2010. 00477.x.

35. Crabb DW, Im GY, Szabo G, Mellinger JL, Lucey MR. Diagnosis and treatment of alcohol-associated liver diseases: 2019 practice guidance from the American Association for the Study of Liver Diseases. Hepatology. 2020;71(1):306-33. https://doi.org/10.1002/hep.30866.

36. Singal AK, Bataller R, Ahn J, Kamath PS, Shah VH. ACG Clinical guideline: alcoholic liver disease. Am J Gastroenterol. 2018;113(2):175-94. https:// doi.org/10.1038/ajg.2017.469.

\section{Publisher's Note}

Springer Nature remains neutral with regard to jurisdictional claims in published maps and institutional affiliations. 